\title{
Real Time Data Communication Using High Altitude Balloon Based on Cubesat Payload
}

\author{
Haklin Kimm, Jin S. Kang, Bob Bruinga, and Ho Sang Ham
}

\begin{abstract}
High-altitude balloons can carry small payloads to altitudes higher than 100,000 feet into the stratosphere to so called "near-space", where satellite components and payloads can be tested in harsh environment to simulate the actual space environment. In particular, a high-altitude balloon flight with satellite communication payloads weighing up to a few pounds is a cost-effective to test the link. In this balloonSat project, several challenges in the stratosphere testing of CubeSat satellite were explored. The focus of the test included functionalities of batteries and $2.4 \mathrm{HGz}$ transceiver in low temperature and long range. In addition, the GPS-enabled radio system functionality was tested for changing altitude and velocity during the flight. A 9 degree-of-freedom sensor logged the flight condition throughout the mission. Using the GPS and Automatic Position Reporting System (APRS), a balloonSat was tracked and recovered successfully. The results showed that all components tested functioned well.
\end{abstract}

Index Terms - Balloon satellite, payload design, high-altitude balloon, space systems software.

\section{INTRODUCTION}

The atmosphere surrounding the Earth's surface consists of several layers, which are defined by physical properties mostly: temperature, wind speed, air density, and atmospheric pressure. The layers of the atmosphere are named, starting from Earth's surface, such as troposphere, stratosphere, mesosphere, thermosphere and exosphere [1]-[3].

The troposphere layer extends from the earth's surface up to 10 to $18 \mathrm{~km}$ in altitude, but the defining altitude varies upon seasons and geographical positions. The air pressure of higher points of the troposphere is measured merely as ten percent of sea level's air pressure. The air temperature of the troposphere generally decreases as it goes higher; and almost all weather phenomena including rain occur in the lower part of this layer.

It is known that around $80 \%$ of the total air mass resides in the lower altitude of the troposphere layer. The stratosphere, which resides above the troposphere, stretched with an altitude of approximately $50 \mathrm{~km}$ above the ground. The ozone layer resides in the upper stratosphere and more than $99 \%$ of the total air mass is concentrated in the first $40 \mathrm{~km}$ from the

Manuscript received January 20, 2015; revised July 6, 2015.

Haklin Kimm is with the Computer Science Department, East Stroudsburg University, East Stroudsburg, PA, USA (email: hkimm@esu.edu).

Jin S. Kang and Bob Bruinga are with Aerospace Engineering Department, U.S. Naval Academy, Annapolis, MD, USA (email: kang@usna.edu and bruninga@usna.edu).

Ho Sang Ham is with the Software Convergence Division, Electronics Telecommunication Research Institute, Daejeon, Korea (email: hham@etri.re.kr). earth's surface. This layer is primarily responsible for absorbing the ultraviolet radiation from the Sun. The stratosphere is characterized by its high static stability, with which the temperature goes up but the pressure decreases as its altitude is higher in this layer [1], [2], [4], [5]. Fig. 1 shows a graphical representation of these regions.

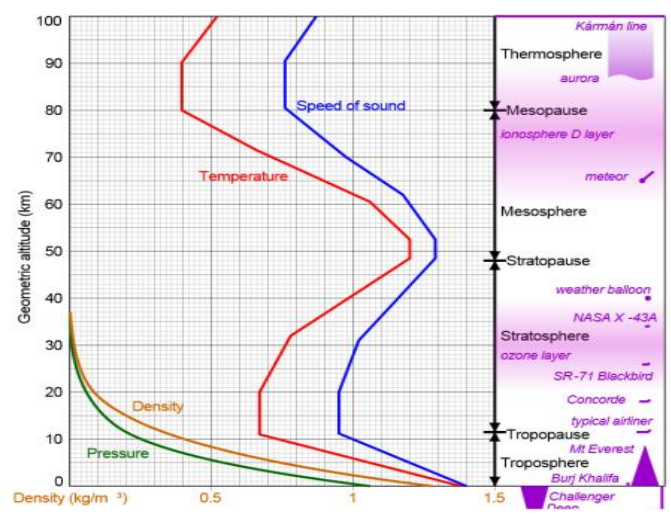

Fig. 1. Atmosphere upon increasing altitude [3].

For this balloonSat project, the main objective was to test CubeSat satellite hardware in stratosphere for their survivability and functionality. Firstly, the components inside the CubeSat satellite [6] were to be tested as a payload to the balloon. Secondly, the satellite communication system consisting of a wireless $2.4 \mathrm{GHz}$ modem (MXH2040 [7]) for long-range signal strength and functionality using a circular patch antenna [8]. In order to verify the full functionality of the $2.4 \mathrm{GHz}$ wireless modem communication with another wireless modem on the ground, which has been connected to a laptop with a wireless "rubber-ducky" antenna, a space system programs that can collect data every second during the balloonSat flight has been developed in-house. Thirdly a 9-degree sensor has been deployed as part of the payload system in order to analyze the balloonSat flight path. The objective was to utilize the $2.4 \mathrm{GHz}$ modem in the stratosphere for transmitting 9-degree sensor data at regular intervals. Accordingly, the ground receiver modem of the laptop computer has been programmed to receive data every second. Lastly, the collected data was to be analyzed in order to build a payload that can be more stable and transmit data with less number of interrupts during the balloon launch and flight [1], [3], [6].

The paper is organized as follows. In Section II, balloonSat design and its related challenges are illustrated. In Section III, the design and development of the balloonSat payloads are described in detail. In Section IV, the tracking of the balloon is illustrated with figures. In Section $\mathrm{V}$, the collected balloonSat data in real time during the flight is explained. Lastly, the paper concludes with summary and future thoughts on the balloonSat. 


\section{BALLOONSAT DESIGN}

A typical balloonSat employed by the East Stroudsburg University weighs up to around $4 \mathrm{~kg}$ and soars up to reach altitudes of higher than $100,000 \mathrm{ft}$ a high altitude balloon and costs only several hundred dollars. This altitude of the stratosphere provides harsh conditions of tough and significant scientific and engineering challenges. At this altitude, temperatures drops to below $-40^{\circ} \mathrm{C}$ and air pressures reach near vacuum conditions as shown in Fig. 1. At this altitude, the balloon expands to about $25 \mathrm{ft}$ in diameter because of the reduced air pressure and eventually bursts on its own. Following the balloon burst, the ballooSat deploys a parachute that allows the payload to survive its trip back to the Earth. When the balloon bursts, initial descent speeds reach the speed of sound $340.3 \mathrm{~m} / \mathrm{s}$. When the balloonSat reaches lower altitudes, condensation can be severe. As seen in Fig. 2, the payload system was contained in a Styrofoam box, into which insulation peanuts were added to make sure the battery temperature does not fall below the specified working temperature. A circular $2.4 \mathrm{GHz}$ patch antenna was placed at the bottom of the balloon payload, as can be seen as a green shiny plate in the figure. A GoPro camera [9] was tightly taped to bottom of the box. A TinyTrak tracking device that uses GPS and Automatic Position Reporting System (APRS) [10] was tethered to the strings that wrap the Styrofoam box, above which a parachute was fastened with the strings hanging just below the balloon. The balloonSat can be tracked throughout its flight and recovered after landing using APRS. A 1200g latex balloon was used for the flight. Fig. 2 shows the remains of the balloon after it burst at high altitude, upon recovery [11]-[14].

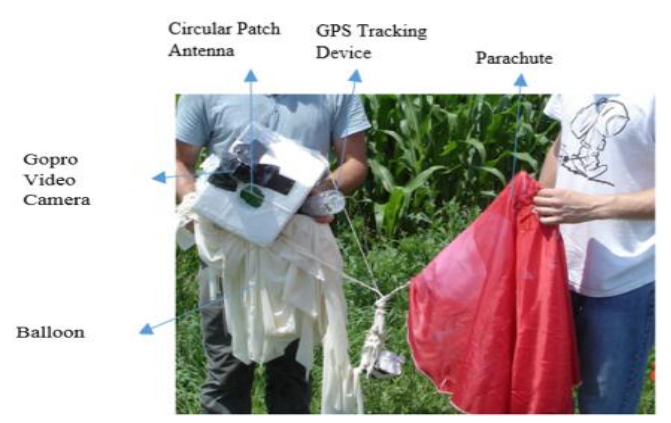

Fig. 2. BalloonSat with payloads procedure for paper submission.

The payload system is based on the Pumpkin CubSat kit with a dsPIC33 pluggable processor module [15], which takes data from a Sparkfun 9 DOF Inertial Measurement Unit that is comprised of three individual chips collecting 3 DOF sensor data respectively. Other satellite components integrated into the CubeSat satellite payload include a Microhard MHX 2420 modem that transmits the string values collected from each sensor over a single UART connection, a $2.4 \mathrm{GHz}$ Flytron circularly-polarized patch antenna [8] that sends signals to the ground receiver, and a Pumpkin Electrical Power Systems (EPS) that uses two 3.7 V Lithium ion batteries for supplying power [6], [16].

The payload system flight software has been developed using $\mathrm{C}$ program language running on a Microchip dsPIC33 [15]. A 16-bit digital signal controller (PPM D2) mounted on top of FM430 Flight Module [6] works with $+5 \mathrm{~V}$ single supply, 3.3V I/O, and stackable 104-pin CubeSat Kit Bus connectors. The flight microcontroller is a TI's single-chip 16-bit MSP430 ultralow-power RISC microcontroller with 50-60KB Flash, 2-10KB RAM, 48 I/O pins, 2 USART, 2 SPI, 1 I2C, 12-bit ADC, 12-bit DAC, 3 DMA, multiple timers, on-board temperature sensor, and multiple clock sources.

The Sparkfun 9DOF sensor node is mounted on top of the CubeSat Kit proto board. The payload node periodically measures three 3 DOF sensor data and transfers the data to the MXH 2420 modem for transmitting to the ground receiver computer. It requires $3.3 \mathrm{~V}$ power, making the interface with the dsPIC processor easy due to the fact that all signal voltages are the same. The Sparkfun 9 DOF Razor uses the Universal Asynchronous Receiver Transmitter (UART) Bus to connect to the dsPIC processor. The 9DOF Razor incorporates three sensors - an ITG-3200 (MEMS triple-axis gyro), ADXL345 (triple-axis accelerometer), and HMC5883L (triple-axis magnetometer) that provides nine degrees of inertial measurement [17]-[19]. The outputs of all sensors are processed by an on-board ATmega328 and transmitted over a serial interface.

\section{BALLOONSAT TRACKING}

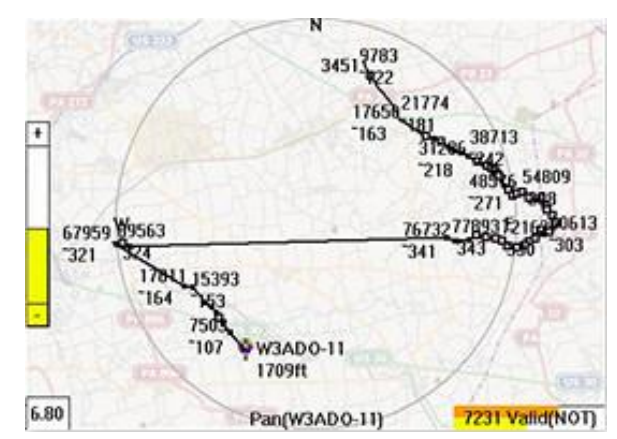

Fig. 3. Tracking path with altitudes.

The tracking device hanging below the balloonSat broadcasts its current GPS coordinates through a satellite link every several minutes. The GPS data points of the balloon path are then recorded, and displayed on the map as shown in Fig. 3. This allows us to track the balloon nearly in real time from the initial launch to the final descent down to the ground, except around more than 5 min with no communication that occurred around the altitude of 77,000 ft. The contact was reestablished around $89,000 \mathrm{ft}$ again on its way down, as in the middle of Fig. 3 as a straight horizontal line crossing over the circle. Fig. 3 also verifies that there has been no communication between altitudes from $76,732 \mathrm{ft}$ to $89,563 \mathrm{ft}$, which has been traced by a ground amateur radio station. This is due to the wrong type of GPS antenna being installed in the balloonSat. A special type of GPS antenna with altitude restriction removed was to be used for the flight, but was switched out by mistake during the assembly of the payload. The "normal" GPS antenna that was used had an altitude limiter that triggered around $88,000 \mathrm{ft}$ of altitude, where the GPS data stopped being delivered. As the payload started to decend, the signal was reestablished as it passed below about $88,000 \mathrm{ft}$ of altitude. Fig. 4 represents a total coverage area that the balloonSat has been detected by amateur radio stations, and that expands more than 325 miles in radius. The balloonSat signals were even detected by some radio stations 
distanced more than $350 \mathrm{mi}$ away. The tracking device of our balloonSat continued to broadcast its position after landing which facilitated locating and retrieval of the balloonSat intact easy. The recovered balloonSat is shown in Fig. 2.

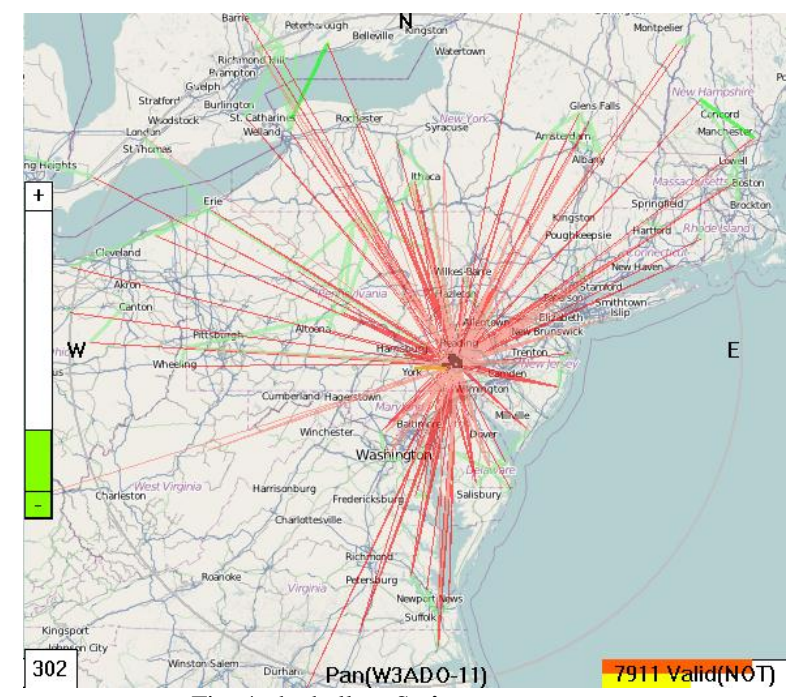

Fig. 4. the balloonSat's coverage area.

\section{REAL Time BALlOONSAT DATA}

During the balloonSat's ascent, the 9 DOF sensor data were collected: 3 DOF of accelerometer (ADXL345), gyroscope (ITG 3200), and magnetometer (HMC5883L). The 9 DOF data had been downlinked, and saved on a ground laptop computer every second during the whole flight. The 9 DOF data had been collected as immediately as the balloonSat starting to lift until it landed on the ground. A part of the downlinked sample data is shown in Fig. 5. Each row starts with the current date, which is followed by 3 DOF values of accelerometer, gyroscope, and magnetometer.

The accelerometer, ADXL345, works with a 10-bit value, and each increment from the Least Significant Bit (LSB) is equivalent to $39 \mathrm{mg}$ or $0.039 \mathrm{~g}$. The ADXL345 is a small, thin, low power, 3-axis accelerometer with high resolution measurement at up to $\pm 16 \mathrm{~g}$. It measures the static acceleration of gravity in tilt-sensing applications, as well as dynamic acceleration resulting from motion or shock. Its high resolution ( $4 \mathrm{~m} g / \mathrm{LSB})$ enables measurement of inclination changes less than $1.0^{\circ}$. Digital output data is formatted as 16-bit twos complement and is accessible through either a SPI (3- or 4-wire) or I2C digital interface. As an example, at uptime 3,256 a $(x, y, z)$ of the ADXL345 is given as $(46,-256$, $-95)$, which is calculated by multiplying $39 \mathrm{mg}$ such as $(46 \times 0.039,-256 \times 0.039,-95 \times 0.039)$ that is equal to $(1.79$, $-9.98,-3.71)$ [18].

The gyroscope, ITG-3200, is a single-chip, digital-output, and 3-axis Microelectromechanical systems (MEMS) gyro Integrated Circuit (IC). The ITG-3200 features three 16-bit analog-to-digital converters (ADCs) for digitizing the gyro outputs, a user-selectable internal low-pass filter bandwidth, and a Fast-Mode I2C (400kHz) interface. Additional features include an embedded temperature sensor and a $2 \%$ accurate internal oscillator. Its digital-output $X-, Y$-, and Z-Axis angular rate sensors (gyros) on one integrated circuit with a sensitivity of $14.375 \mathrm{LSBs}$ per \% and a full-scale range of $\pm 2000 \%$ s [17].
The magnetometer, HMC5883L is a surface-mount, multi-chip module designed for low-field magnetic sensing with a digital interface for applications such as low-cost compassing and magnetometers. The HMC5883L provides a 12-bit ADC that enables $1^{\circ}$ to $2^{\circ}$ compass heading accuracy. The I2C serial bus allows for easy interface. This sensor features precision in-axis sensitivity and linearity, and is designed to measure both the direction and the magnitude of Earth's magnetic fields, from millimeter-gauss to 8 gauss [19]. Fig. 5 shows a sample output of the collected 6 DOF data.

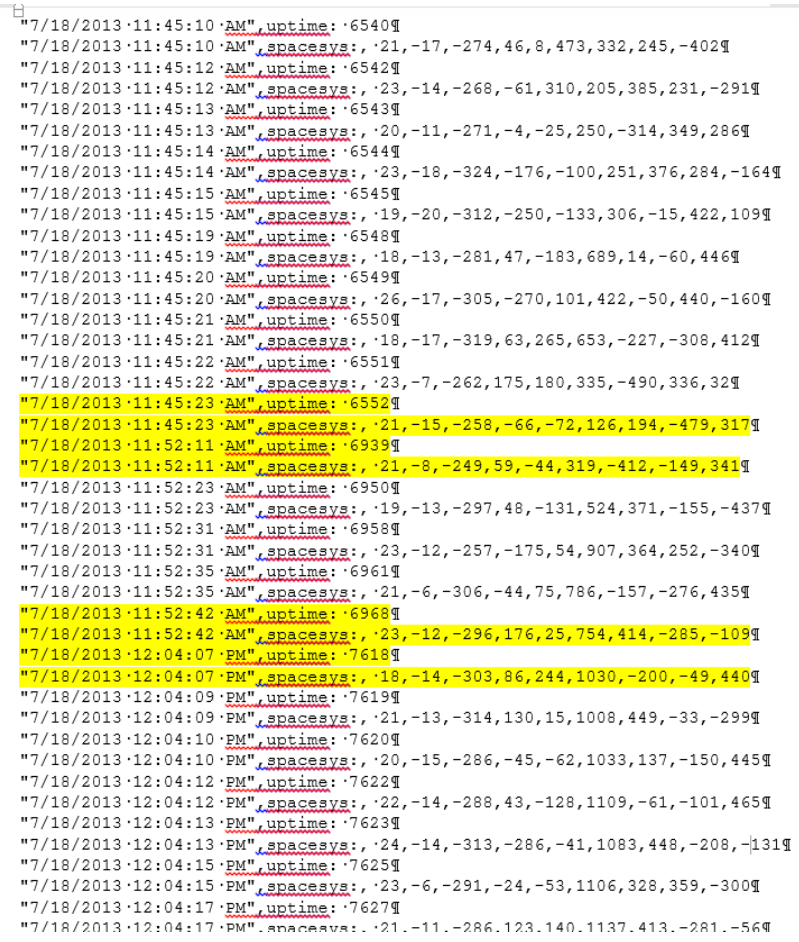

Fig. 5. 9DOF sensor data collected during the no contact.

\section{BALloOnSAT Data ANALYSiS}

Approximately 5,000 data elements have been collected in real-time during the flight that started at a relative time of 3,240 and ended at 8,348 , which is equivalent to around $5,100 \mathrm{~s}$ as each data point was collected every second. Note that the receiver computer for the project has been programmed to collect 9 DOF sensor data every second in real time during the balloon flight, so as to collect the data for more than one-and-half hours which was almost the whole flight time except the last several minutes' of high-velocity landing over the slight hill of corn fields. It was challenging to align the receiver antenna with the balloonSat antenna because the line of sight was difficult to maintain during the landing.

A further investigation into the period of loss-of-contact has been carried out by examining the sensor data collected during this period of time. The sensor data collected before and after the no-data periods are applied for further analysis, which have been highlighted in Fig. 5 as shown. Upon analyzing the gyroscope data of z-axis, there had been much turbulence during the flight. Based upon the accelerometer data of z-axis, the balloonSat had been ascending and descending at a steady pace. Those data sets are plotted in Fig. 6 using MATLAB [20]. The highlighted data sets indicate 
that there have been no data collected from the balloonSat. The first instance of no data was happened between the time unit 6,552 and 6,939, which translates to approximately at 7 min mark, and the last instance of no data was between time 6,968 and 7,618, which would be approximately at $10 \mathrm{~min}$ mark. As shown in Fig. 6, the data have been plotted upon the corresponding time units so that the time unit zero of $\mathrm{x}$-axis is mapped to the time unit 6,445 from the entire sensor data set. The data at unit 107 of $x$-axis in Fig. 6 is the data collected just before the data collection gap from the balloonSat, as well as the data at unit 113. Based on the analysis, the data collection gap can be attributed to higher-than-normal acceleration occurrence at around time unit of 113, as shown by high fluctuation recorded by the $\mathrm{z}$-axis accelerometer.

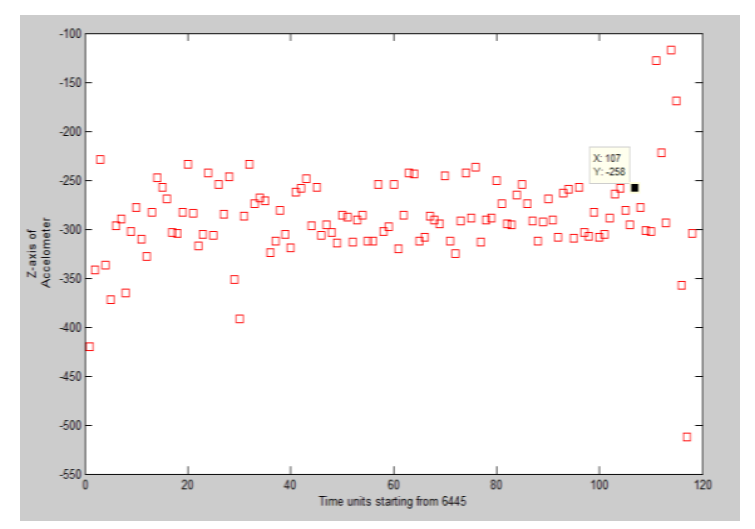

Fig. 6. Accelerometer data collected during the no contact.

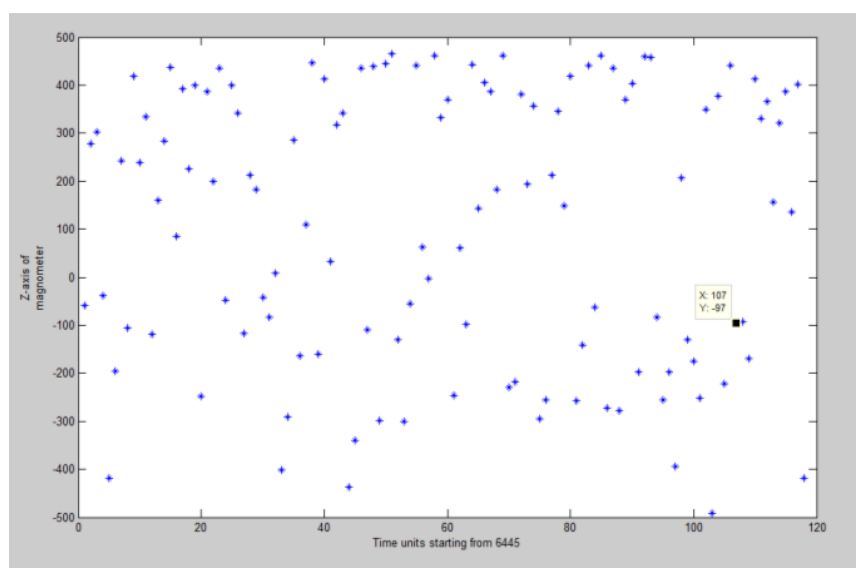

Fig. 7. Magnetometer data collected during the no contact.

Based on analysis the no-data periods would have occurred due to sudden changes in wind speed. In order to see this plausibility of no-data, magnetometer data has been selected for the analysis. Fig. 7 shows the z-axis data of magnetometer plotted upon the corresponding time unit. As seen in the above plot, however it is not easy to say that there exist some differences around the time unit 107 or 113 since most data have been showing no noticeable differences in magnetometer data. In addition to the reasoning mentioned above, another possibility of stoppage in 9 DOF data collection can be due to a very high speed and gusty wind conditions that moved that balloon payload more than $21 \mathrm{mi}$ in about 5 min between two locations: (40 $185695 \mathrm{~N}, 7621$ $4855 \mathrm{~W}$ ) and (40 $201515 \mathrm{~N}, 76004046 \mathrm{~W}$ ) - (latitude, longitude). As can be seen in the position data shown in Fig. 3, there is almost no change in altitude value but there is a difference of $21 \mathrm{mi}$ of longitude, which is approximately equivalent to $21 \mathrm{mi}$ on earth surface. From the analysis of data, it can be deduced that the balloon travelled approximately $21 \mathrm{mi}$ in $5 \mathrm{~min}$, which means that the average velocity of the balloon during this time is $250 \mathrm{mi} / \mathrm{hr}$. If the balloon travelled from east to west with this speed of 250 $\mathrm{mi} / \mathrm{hr}$, the linear circular antenna of the balloonSat may not be pointed correctly to nadir direction due to high accelerations such that the communication could not be established with the receiver on the earth ground.

\section{CONCLUSION}

High-altitude balloon satellite cost is relatively low but still provides a good testing platform for near space experiments as the conditions provided at high altitudes are similar to development and operation of real spacecraft systems. In this project, an actual CubeSat satellite hardware, as fully integrated into the CubeSat frame, was tested for functionality at the extreme environments encountered in the stratosphere. The results showed that the payload built with a CubeSat frame worked well during the entire flight as planned, and the wireless $2.4 \mathrm{GHz}$ modem mostly transmits signals well every second to the ground station receiver connected to the laptop computer with a wireless "rubber-ducky" antenna. The flight software that was developed in-house for the balloonSat worked well during the whole balloonSat flight in collecting the 9 DOF sensor data in real time. The 9-degree sensor deployed as part of the balloonSat worked well to enable collection of the data for analyzing the balloonSat flight pattern. However, the data showed that there was a gap in the sensor data during the flight. When the balloonSat was at a higher altitude, the ground receiver could not receive the signals for more than 5 min from the $2.4 \mathrm{GHz}$ modem integrated into the balloon payload. From analysis, it is likely that a possible high-speed wind of $250 \mathrm{mi} / \mathrm{hr}$ may be the cause of this loss-of-contact. The attached antenna may have been swinging off-nadir due to high accelerations. This phenomenon will be tested further in subsequent balloon launches at the same location.

The collected data have been analyzed in order to build a better payload system that can be more stable and transmit data with less number of interrupts during the balloon launch and flight. The stabilization of the balloonSat platform is to be improved in order to achieve more efficient experiment process, as well as for safety to the hardware and recovery of the paylaod. The collected data from the accelerometer and gyro sensors are analyzed to characterize the stability of the payload during the flight. In the immediate future, our current payloads shall be upgraded to accommodate live telemetry through packet radio broadcasts as well as $2.4 \mathrm{GHz}$ wireless modem. This update of the payloads will demand more experimentation on range and battery power usage, and stable antenna system that can transmit data continuously. It is expected to build and test the stratospheric communication systems operating in the higher frequencies as well as 2.4 $\mathrm{GHz}$, which are very susceptible to harsh atmosphere.

\section{ACKNOWLEDGMENT}

We are very grateful to the students, who participated in this project, of Computer Science Department at East 
Stroudsburg and Aerospace Engineering Department at the U.S. Naval Academy.

\section{REFERENCES}

[1] J. R. Wertz et al., Space Mission Engineering: The New SMAD, 1st ed. California, USA: Microcosm Press, 2011.

[2] D. Grace and M. Mohorcic, Broadband Communications via High-Altitude Platforms, John Wiley \& Sons Publishing, 2011.

[3] U.S. Standard Atmosphere Report, "Geometric altitude vs. temperature, pressure, density, and the speed of sound," 1962.

[4] J. M. Park et al., "Technology development for wireless communications system using stratospheric platform in Korea," in Proc. the 13th IEEE International Symposium on Personal, Indoor and Mobile Radio Communications, Daejeon, Korea, 2012, vol. 4, pp. 1577-1581.

[5] G. Kandus et al., "Modelling of atmospheric impairments in stratospheric communications," in Proc. the 2nd WSEAS Int. Conf. On Circuits, Systems, Signal \& Telecommunications Acapulco, Mexico, 2008.

[6] H. Kimm, "Interdisciplinary capstone group project: CubeSat system development in a small-sized institution," in Proc. the International Conference on New Perspectives in Science Education, Florence, Italy, 2013.

[7] Wireless Satellite Modem. (2012). [Online]. Available: http://www.microhardcorp.com/Spectra2420.php

[8] Circular Patch Antenna. (2012). [Online]. Available: www.flytron.com/osd-headtrackers/53-11db-patch-antenna.html

[9] Video Camera. (2012). [Online]. Available: http://gopro.com/cameras

[10] GPS Position Encoder. (2012). [Online]. Available: http://www.byonics.com/tinytrak4/

[11] R. Karol and J. Lee, "High altitude ballooning for space and atmospheric observation," Caltech High Altitude Balloon Report, 2012.

[12] C. Koehler, "BalloonSat: Missions to the edge of space," in Proc. the 16th Annual/USU Conference on Small Satellites, Logan, Utah, USA, 2004.

[13] J. Flaten, "Using high-altitude ballooning to give freshmen a hands-on introduction to the space side of aerospace," in Proc. $120^{\text {th }}$ ASEE Annual Conference \& Exposition, Atlanta, GA, 2013.

[14] D. Blandford, "Tracking high altitude balloons in an EE projects class," in Proc. $120^{\text {th }}$ ASEE Annual Conference \& Exposition, Atlanta, GA, 2013.

[15] T. Wilmshurst, Designing Embedded Systems with PIC Microcontrollers, $2^{\text {nd }}$ ed. Oxford, UK: Newnes Publishing, 2010.

[16] D. Thiel et al., "Monitoring stick speed and ball control in field hockey drills using a stick-mounted inertial accelerometer," Procedia Engineering, vol. 34, pp. 574-579, 2012.

[17] Gyroscope Triple Axis. [Online]. Available: https://www.sparkfun.com/products/9793

[18] Accelerometer. [Online]. https://www.sparkfun.com/products/9045

[19] Magnetometer. [Online]. https://www.sparkfun.com/products/10494

[20] Gonzalez et al., Digital Image Processing Using MATLAB, $2^{\text {nd }}$ ed. USA: Gatesmark Publishing, 2009.

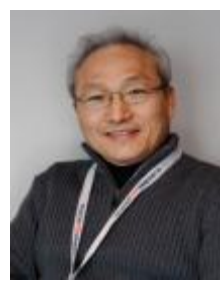

Haklin Kimm received his M.S. and Ph.D. degrees in computer science from the Department of Electrical Engineering and Computer Science at University of Oklahoma. Currently he is a professor in the Computer Science Department at East Stroudsburg University of Pennsylvania, East Stroudsburg in USA, where he teaches computer architecture, networks and operating systems. His major research interests are in the area of automotive software engineering, image processing, distributed and parallel systems, multilevel security with SELinux. Currently he works on Balloon Satellite Payload Development, CAN Bus Networks, Multilevel Security with SELinux, Edge Detection and Linking. He also leads Integrated CubeSAT Bus System Project with the Space Systems Software Lab., Computer Science Department at East Stroudsburg, PA, where PocoSAT1 and PocoSAT2 had been built in 2012. He had been invited to be an exchange visiting faculty from University of Guam, Korea University and Hirosaki University, Japan. He gave talks and tutorials at various institutions and research centers, i.e., Samsung SDS and ETRI in Korea. He has experiences doing research at Oak Ridge National Lab., NASA, and ETRI.

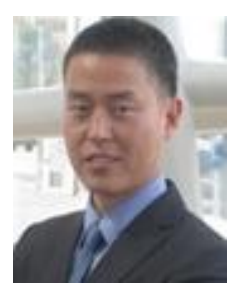

Jin S. Kang received his B.S. degree from University of Michigan, received his M.S. degree from Stanford University, and his Ph.D. degree from Korea Aerospace University, all in aerospace engineering. After working for General Electric for two years, Kang served in Korea Air Force Academy as a professor while holding a rank of $1^{\text {st }}$ LT. His main research area is in space systems engineering and was involved in development of four micro-satellites and 6 CubeSat satellites. He is currently an assistant professor (civilian) in the Aerospace Engineering Department at the United States Naval Academy, and serves as the director of the Naval Academy Small Satellite Program.

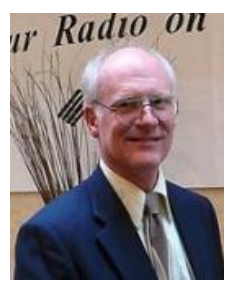

Bob Bruninga received his BSEE degree from Georgia Tech. in 1970 and MSEE degree from the Navy PG School in Monterey. He served a career as an engineering duty officer in the US Navy and later as a civilian has served as an instructor and as an senior research engineer in the Aerospace Department at the US Naval Academy. In 1993 he developed the APRS protocol which is used for tracking most amateur balloon payloads. He has also developed 7 small satellites operating in the Amateur Satellite Service supporting worldwide relay of APRS signals.

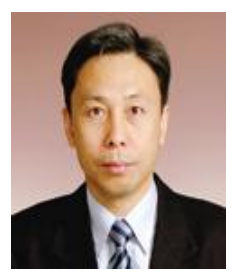

Ho Sang Ham received his M.S. and Ph.D. degrees in industrial engineering from Korea University. Currently he leads the Creative Strategy and Planning Division of Electronics and Telecommunications Research Institute, Daejon, where he takes the lead in creating a new driving force for the innovative future of the society by identifying customer and industry needs, national $R \& D$ policies and creative strategies and planning. Before serving as the vice president, he had been actively working on research areas related to robotics, embedded systems, automotive software engineering, and field bus application to ship building as a project leader. His contribution on software technology in Korea has been acknowledged by the president and the minister of information and technology. He serves as the vice president for Korea Embedded Systems Association, the president for Embedded Software Technology Forum, a board member for Korea Defense Software, and an editorial member for Journal of Communications, Korea. 